\title{
The risk of sarcopenia 24 months after bariatric surgery - assessment by dual energy X-ray absorptiometry (DEXA): a prospective study
}

\author{
Matej Pekař ${ }^{1,2}$, Anna Pekařová2 ${ }^{2}$ Marek Bužga ${ }^{3}$, Pavol Holéczy ${ }^{1,4}$, Marek Soltes ${ }^{5}$ \\ ${ }^{1}$ Department of Surgery, Vítkovice Hospital INC, Ostrava, Czech Republic \\ ${ }^{2}$ Department of Physiology, Faculty of Medicine, Masaryk University, Brno, Czech Republic \\ ${ }^{3}$ Department of Human Movement Studies, Faculty of Education, University of Ostrava, Ostrava, Czech Republic \\ ${ }^{4}$ Department of Surgical Disciplines, Faculty of Medicine, University of Ostrava, Ostrava, Czech Republic \\ ${ }^{5} 1^{\text {st }}$ Department of Surgery, University of Pavol Jozef Safarik, Kosice, Slovak Republic
}

Videosurgery Miniinv 2020; 15 (4): 583-587

DOI: https://doi.org/10.5114/wiitm.2020.93463

\begin{abstract}
Introduction: Bariatric procedures lead to changes in body composition. Desired fat loss may be accompanied by decrease of muscle mass, thus raising the risk of sarcopenia.

Aim: To detect the risk of sarcopenia in patients 24 months after different bariatric/metabolic (B/M) procedures by DEXA.

Material and methods: Consecutive patients scheduled for a B/M procedure underwent DEXA scan and anthropometric assessment before and 24 months after surgery in a prospective manner. Obtained data were tested for significant differences $(p<0.05)$ to detect body composition changes and occurrence of sarcopenia. The International Physical Activity Questionnaire (IPAQ) was answered at 24 months to assess physical activity.

Results: Nineteen patients were enrolled, with no drop-off at follow-up. Body mass index dropped from $42.4 \pm 6.3$ to $30.3 \pm 4.9 \mathrm{~kg} / \mathrm{m}^{2}$, with excess weight loss of $72 \pm 25 \%$ and substantial improvement of all relevant anthropometric measurements $(p<0.001)$. Significant changes in DEXA parameters were observed: fat mass index $(19.5 \pm 4.7 \mathrm{vs}$. $\left.12.1 \pm 3.7 \mathrm{~kg} / \mathrm{m}^{2}\right)$, estimated visceral adipose area $\left(235.8 \pm 70.0 \mathrm{vs} .126 .5 \pm 50.4 \mathrm{~cm}^{2}\right)$, lean mass index $(22.1 \pm 2.4 \mathrm{vs}$. $\left.18.1 \pm 2.3 \mathrm{~kg} / \mathrm{m}^{2}\right)$, appendage lean mass index $\left(9.7 \pm 1.3 \mathrm{vs} .7 .7 \pm 1.1 \mathrm{~kg} / \mathrm{m}^{2}\right)$, bone mineral content $(1.22 \pm 0.1 \mathrm{vs} .1 .12$ $\pm 0.1 \mathrm{~kg}$ ), Z score (2.32 vs. 0.96) and T score (0.58 vs. -0.58 ). A low level of physical activity was recorded at 24 months. Conclusions: $B / M$ procedures lead to significant changes in body composition at 24 months after surgery. DEXA detects these changes effectively. Desired fat loss is associated with significant reduction of skeletal muscle and bone mineral mass. As such, patients after B/M surgery are at risk of sarcopenia. A low level of physical activity may also play a negative role.
\end{abstract}

Key words: obesity, bariatric surgery, laparoscopy, metabolic surgery, sarcopenia, dual-energy X-ray absorptiometry.

\section{Introduction}

The evolving obesity pandemic has become a burning issue worldwide. Nowadays, obesity is being considered a chronic disease with serious health consequences affecting the cardiovascular system (hypertension, ischaemic heart disease), metabolism of glucose, lipids and minerals (diabetes, hyperlipidaemia with subsequent atherosclerosis), the respi-

\section{Address for correspondence}

Assoc. Prof. Marek Soltes MD, PhD, $1^{\text {st }}$ Department of Surgery, University of Pavol Jozef Safarik, Tr. SNP 1, 04190 Kosice, Slovak Republic, e-mail: soltes.marek@yahoo.com 
ratory (sleep apnoea) and musculoskeletal system (arthrosis), as well as mental health (depression), resulting in shortened life expectancy and decreased quality of life. Also of note, obesity leads to impaired fertility and adversely affects pregnancy [1, 2]. The prevalence of obesity worldwide has nearly tripled since 1975. According to the World Health Organisation (WHO), 39\% of adults (aged 18 years and more) were overweight in 2016, while $13 \%$ were obese [3].

Although a conservative approach is usually the first choice therapeutic option for obese patients, it remains associated with a high failure rate in the long term, due to rebound weight gain resulting in a vicious circle. As such, bariatric/metabolic (B/M) surgery represents the most effective sustainable treatment option for the time being [4-6]. Several bariatric procedures were described and proven effective, such as laparoscopic sleeve gastrectomy (LSG), Roux-en-Y gastric bypass (RYGB) and laparoscopic greater curvature plication (LGCP), which recently gained significant popularity in the Czech Republic. Other surgical methods were reported and utilised as well, but have not gained wider implementation. The surgical approach to obesity favours a laparoscopic approach due to well-documented advantages, including faster recovery, decreased postoperative pain, fewer surgical-site infections and diminished wound complications [7].

Effective $\mathrm{B} / \mathrm{M}$ procedures may lead to changes in body composition. Desired fat loss is often accompanied by a decrease of muscle mass as well, which may be so significant that the patients are at risk of sarcopenia development [8]. Dual-energy X-ray absorptiometry (DEXA) offers the best reproducibility and accuracy to detect such changes $[9,10]$. Some authors advocate the need for preventive programmes to overcome the problem of sarcopenia [11], such as the combination of aerobic and power exercises, which appears to be the most effective approach [12-14].

\section{Aim}

The aim of the study is to detect the occurrence of sarcopenia in patients 24 months after different $\mathrm{B} / \mathrm{M}$ procedures by DEXA.

\section{Material and methods}

Consecutive patients scheduled for a B/M procedure due to obesity were enrolled in the prospective study approved by the Multicentre Ethics Committee of Vitkovice Hospital (ethical approval number: EK/234/2018), after obtaining their informed consent. Indications for surgery, based on $\mathrm{BMI}$ and comorbidities (7x arterial hypertension, $4 \times$ type 2 diabetes, $4 \times$ hyperlipidaemia), were approved by the multidisciplinary bariatric team, following the guidelines of the International Federation for the Surgery of Obesity (IFSO) [15], while respecting personal preference of the patients with regards to the type of procedure. All procedures were aimed to be performed laparoscopically, applying previously described standard techniques $[16,17]$.

DEXA measurements were performed before and 24 months after the B/M procedure using the Hologic Discovery A densitometer (Mississauga, Ontario, Canada), calibrated according to the manufacturer's recommendations. The precision error and coefficient of variation were established, which allowed the value of the minimal significant change to be calculated as described in previous studies [18-20]. Recorded measurements included fat mass index (FMI) - a measure of relative fat content, independent of fat free mass, estimated visceral adipose tissue (Est VAT) - estimated amount of visceral fat, lean mass index (LMI) - a measure of relative lean content, independent of fat mass, appendage lean mass index (ALMI) - a measure of relative lean mass in arm and legs, bone mineral content (BMC), $Z$ score - for patients aged under 50 and $T$ score - for those above 50 as measures of osteoporosis. Furthermore, every patient underwent anthropometric assessment which included a record of body weight, height, body mass index (BMI), excess body weight (EBW) calculated over BMI $25 \mathrm{~kg} / \mathrm{m}^{2}$, waist and hip circumference, as well as biceps, triceps, supraspinatus and subscapular skinfold. Excess weight loss (EWL) was calculated as the percentage of weight loss to EBW. To assess physical activity after surgery, the International Physical Activity Questionnaire (IPAQ) was answered at 24 months [21].

\section{Statistical analysis}

Obtained data were tested for significant differences using licensed software (Statistica 12, StatSoft, CZ). The Wilcoxon signed rank test was used as the analysed data did not follow a normal distribution. Differences were considered statistically significant for a $p$-value under 0.05 .

\section{Results}

Nineteen patients (4 males and 15 females, mean age: $46.3 \pm 8.9$ years), after different $B / M$ procedures 
Table I. Anthropometric data at baseline and after 24 months

\begin{tabular}{|c|c|c|c|}
\hline Parameter $[\mathrm{cm}]$ & Baseline & After 24 months & $P$-value \\
\hline Waist circumference & $129 \pm 16$ & $93 \pm 11$ & $<0.001$ \\
\hline Hip circumference & $128 \pm 13$ & $107 \pm 11$ & $<0.001$ \\
\hline Triceps skinfold & $4.8 \pm 0.7$ & $2.6 \pm 0.8$ & $<0.001$ \\
\hline Supraspinatus skinfold & $4.3 \pm 1.1$ & $2.5 \pm 0.9$ & $<0.001$ \\
\hline Biceps skinfold & $3.8 \pm 1.0$ & $1.9 \pm 0.6$ & $<0.001$ \\
\hline Subscapular skinfold & $4.5 \pm 1.1$ & $2.7 \pm 0.8$ & $<0.001$ \\
\hline
\end{tabular}

(3× LGCP, 15× LSG, $1 \times$ RYGB), were enrolled in the study with no drop-off at follow-up at 24 months. Mean BMI dropped from $42.4 \pm 6.3$ to $30.3 \pm 4.9 \mathrm{~kg} / \mathrm{m}^{2}$ ( $p<0.001$ ) with mean EWL of $72 \pm 25 \%$. Relevant anthropometric measurements decreased significantly as well (Table I). As for DEXA parameters (Figure 1, Table II), highly significant changes $(p<0.001)$ were observed for FMI, $19.5 \pm 4.7$ vs. $12.1 \pm 3.7 \mathrm{~kg} / \mathrm{m}^{2}$, Est VAT, $235.8 \pm 70.0$ vs. $126.5 \pm 50.4 \mathrm{~cm}^{2}$, LMI $22.1 \pm 2.4$ vs. $18.1 \pm 2.3 \mathrm{~kg} / \mathrm{m}^{2}$, ALMI $9.7 \pm 1.3$ vs. $7.7 \pm 1.1 \mathrm{~kg} / \mathrm{m}^{2}$, BMC $1.22 \pm 0.1$ vs. $1.12 \pm 0.1 \mathrm{~kg}, Z$ score - for age under 50 (2.32 vs. 0.96) and T score - for age above 50 (0.58 vs. -0.58$)$. The results confirm significant changes in body composition at 24 months after $\mathrm{B} / \mathrm{M}$ surgery, where desired fat loss is clearly associated with a significant decrease of skeletal muscle mass and bone mineral mass as well. Also of note, a low level of physical activity was recorded within the patient group, based on the results of IPAQ at 24 months after surgery (Table III).

\section{Discussion}

$\mathrm{B} / \mathrm{M}$ surgery represents the most effective therapeutic tool for morbid obesity and a promising approach towards obesity-related comorbidities, because, unlike conservative treatment, it assures

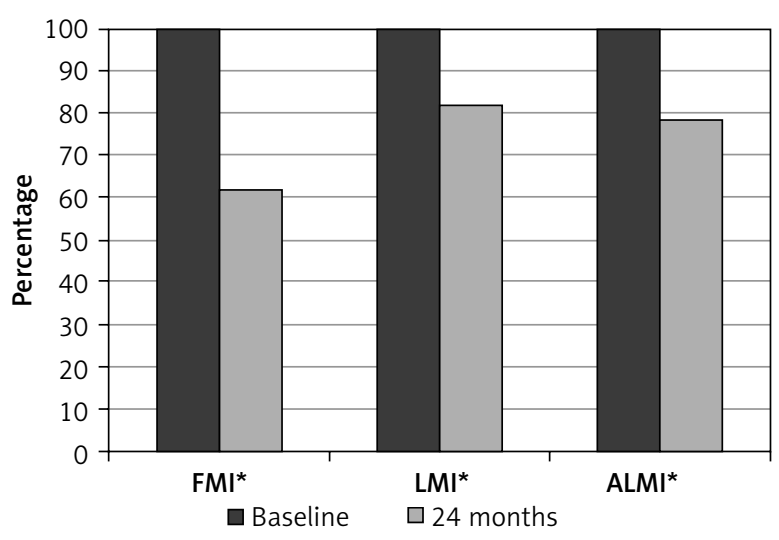

Figure 1. Fat mass index (FMI), lean mass index (LMI) and appendage lean mass index (ALMI) at baseline and after 24 months ${ }^{*}$-value $<0.001$.

significant, quick and relatively sustainable weight loss [19, 22-24]. Although this effect is predominantly based on the desirable fat mass reduction, it may also be associated with a decrease of other body components, such as muscles and bones $[10,19,20]$. Both obesity and sarcopenia increase health-related risks, especially when they co-exist. Therefore, the European Society for Clinical Nutrition and Metabolism (ESPEN) and the European Associa-

Table II. Median FMI, LMI and ALMI at baseline and after 24 months - overall and split by the procedure

\begin{tabular}{|lccccccc|}
\hline B/M procedure & $N$ & $\begin{array}{c}\mathrm{FMI}\left[\mathrm{kg} / \mathrm{m}^{2}\right] \\
\text { Baseline }\end{array}$ & $\begin{array}{c}\mathrm{FMI}\left[\mathrm{kg} / \mathrm{m}^{2}\right] \\
\text { After } \\
24 \text { months }\end{array}$ & $\begin{array}{c}\mathrm{LMI}\left[\mathrm{kg} / \mathrm{m}^{2}\right] \\
\text { Baseline }\end{array}$ & $\begin{array}{c}\mathrm{LMI}\left[\mathrm{kg} / \mathrm{m}^{2}\right] \\
\text { After } \\
24 \text { months }\end{array}$ & $\begin{array}{c}\mathrm{ALMI}\left[\mathrm{kg} / \mathrm{m}^{2}\right] \\
\text { Baseline }\end{array}$ & $\begin{array}{c}\mathrm{ALMI}\left[\mathrm{kg} / \mathrm{m}^{2}\right] \\
\text { After } \\
\text { 24 months }\end{array}$ \\
\hline Overall & 19 & 19.1 & $12.3^{*}$ & 21.3 & $18.1^{*}$ & 9.39 & $7.45^{*}$ \\
\hline LSG & 15 & 19.5 & $11.5^{*}$ & 21.7 & $17.6^{*}$ & 9.58 & $7.45^{*}$ \\
\hline LGCP & 3 & 19.1 & 14.2 & 19.8 & 18.1 & 8.57 & 7.65 \\
\hline RYGB & 1 & 15.3 & 9.2 & 20.2 & 15.8 & 8.89 & 6.57 \\
\hline
\end{tabular}

${ }^{*}$-value $<0.001,{ }^{* *}$ patient with metabolic syndrome. 
Table III. IPAQ - physical activity categories

\begin{tabular}{|lcc|}
\hline $\begin{array}{l}\text { Level of physical } \\
\text { activity }\end{array}$ & $\begin{array}{c}\text { Time of activity } \\
\text { per day }[\mathrm{h}]\end{array}$ & $\begin{array}{c}\text { Number } \\
\text { of patients }\end{array}$ \\
\hline High & $>1.0$ & 0 \\
\hline Moderate & $0.5-1.0$ & 0 \\
\hline Low & $<0.5$ & 19 \\
\hline
\end{tabular}

tion for the Study of Obesity (EASO) call on researchers and clinicians to study and challenge sarcopenic obesity [11].

Although DEXA represents an excellent tool to detect changes in body composition [9, 10], it is used relatively infrequently to assess series of patients after mixed $\mathrm{B} / \mathrm{M}$ procedures with respect to sarcopenia. Taking into account the advantages of this methodology, allowing for clear analysis of the weight loss structure, it should be used more systematically to reveal the true effects of $B / M$ surgery.

The weight and fat loss effect observed in our study was excellent. BMI dropped from class III obesity to class I. Observed Est VAT (representing the fat around internal organs such as heart, lung, liver and others) decreased from the "high risk" (above $160 \mathrm{~cm}^{2}$ ) to "increased risk" category (above $100 \mathrm{~cm}^{2}$ ) for obesity complications $[25,26]$. As for the BMC, values under the normal range were recorded before surgery, with a further decrease at 24 months after the $\mathrm{B} / \mathrm{M}$ procedure. Although $\mathrm{Z}$ and $\mathrm{T}$ scores dropped as well, none of the patients qualified for risk of osteopenia or osteoporosis.

With regards to possible sarcopenia, LMI and ALMI were analysed in our study. While LMI represents the body mass without fat and without bones (includes internal organs), ALMI describes skeletal muscle mass of the extremities (independently of internal organs) - as a substantial number of study population underwent LSG, both LMI and ALMI were considered fundamental for possible sarcopenia detection. Baumgartner et al. described cut-off values for sarcopenia for men and women as 7.26 and $5.45 \mathrm{~kg} / \mathrm{m}^{2}$ respectively [27]. None of our patients crossed over these values, but some were very close to the sarcopenia status at 24 months after surgery. This is an alarming finding that requires further attention of multidisciplinary teams dealing with patients subjected to B/M surgery.

Based on the IPAQ evaluation, a negligible impact of physical activity on weight loss in our study popu- lation was detected. At the same time, lack of physical activity might represent a contributing factor towards observed skeletal muscle mass reduction. To decrease the risk of muscle mass loss, power exercise in combination with aerobic exercise is recommended in some studies [12-14]. Whether this logical assumption translates into clinical practice remains unclear, so further studies are needed with proper design. Based on our findings, a structured rehabilitation programme controlled by physiotherapist is planned to be included in our standard postoperative care after B/M procedures for 24-48 months to assess their impact on sarcopenia development and to decrease the risk of postoperative weight gain.

Despite the statistically significant results, there are some limitations that should be taken into consideration. The sample size of the study population is relatively low, while the spectrum of $B / M$ procedures is relatively wide. As such, our results should be considered as pilot data, drawing attention to the problem of muscle mass loss after B/M procedures in general, which seems to be associated with fat mass loss at 24 months after surgery.

DEXA, which was implemented for assessment of body composition changes after B/M surgery, with special focus on sarcopenia detection, was found to be a reliable, objective tool with a high acceptability rate among the patients (no drop-off from the study population on follow-up at 24 months). As a result, this methodology might be re-applied by other researchers, bearing in mind the financial burden.

\section{Conclusions}

$B / M$ procedures lead to significant changes in body composition at 24 months after surgery. DEXA detects these changes effectively. Although desirable loss of fat mass is the most obvious, reduction of skeletal muscle mass and bone mineral mass is significant as well. As such, patients after B/M surgery can be at risk of sarcopenia. A low level of physical activity, which seems to be characteristic for this patient group, may also play a negative role. Therefore, preventive measures to avoid sarcopenia in patients after $\mathrm{B} / \mathrm{M}$ procedures might be justified.

\section{Acknowledgments}

This study was supported by Specific University Research grant no. MUNI/A/1307/2019 provided by the Ministry of Education, Youth and Sports of the Czech 
Republic. The authors wish to acknowledge the contribution of Mgr. Jitka Macháčková (data collection) and of Martin Bolek, MD. (identification of patients).

\section{Conflict of interest}

\section{The authors declare no conflict of interest.}

\section{References}

1. IFSO. Obesity consequences | obesity problems [Internet]. Available from: https://www.ifso.com/consequences/

2. Kuno T, Tanimoto E, Morita S, et al. Effects of bariatric surgery on cardiovascular disease: a concise update of recent advances. Front Cardiovasc Med 2019; 6: 94.

3. WHO. Obesity and overweight. Available from: https://www. who.int/news-room/fact-sheets/detail/obesity-and-overweight

4. Moya-Ramón M, Picó-Sirvent I, Aracil-Marco A. Effects of physical activity programmes in severe obesity before and after bariatric surgery: a current framework. Eur J Hum Mov 2018; 41: 103-23.

5. Spaniolas K, Kasten KR, Celio A, et al. Postoperative follow-up after bariatric surgery: effect on weight loss. Obes Surg 2016; 26: 900-3.

6. Buzgova R, Buzga M, Holeczy P, et al. Evaluation of quality of life, clinical parameters, and psychological distress after bariatric surgery: comparison of the laparoscopic sleeve gastrectomy and laparoscopic greater curvature plication. Bariatr Surg Pract 2016; 11: 169-76.

7. Reoch J, Motillo S, Shimony A, et al. Safety of laparoscopic vs open bariatric surgery: a systematic review and meta-analysis. Arch Surg 2011; 146: 1314-22.

8. Voican CS, Lebrun A, Maitre S, et al. Predictive score of sarcopenia occurrence one year after bariatric surgery in severely obese patients. PLoS One 2018; 13: e0197248.

9. Tallroth K, Kettunen JA, Kujala UM. Reproducibility of regional DEXA examinations of abdominal fat and lean tissue. Obes Facts 2013; 6: 203-10.

10. Lohman M, Tallroth K, Kettunen JA, et al. Reproducibility of dual-energy $\mathrm{X}$-ray absorptiometry total and regional body composition measurements using different scanning positions and definitions of regions. Metabolism 2009; 58: 1663-8.

11. Barazzoni R, Bischoff S, Boirie Y, et al. Sarcopenic obesity: time to meet the challenge. Obes Facts 2018; 11: 294-305.

12. Dieli-Conwright CM, Courneya KS, Demark-Wahnefried W, et al. Effects of aerobic and resistance exercise on metabolic syndrome, sarcopenic obesity, and circulating biomarkers in overweight or obese survivors of breast cancer: a randomized controlled trial. J Clin Oncol 2018; 36: 875-83.

13. Park J, Kwon Y, Park H. Effects of 24-week aerobic and resistance training on carotid artery intima-media thickness and flow velocity in elderly women with sarcopenic obesity. J Atheroscler Thromb 2017; 24: 1117-24.

14. Gando Y. Carotid artery parameters after combined exercise training in women with sarcopenic obesity. J Atheroscler Thromb 2017; 24: 1090-1.
15. IFSO. Body Mass Index. Available from: https://www.ifso.com/ body-mass-index/.

16. Bužga M, Holéczy P, Švagera Z, et al. Laparoscopic gastric plication and its effect on saccharide and lipid metabolism: a 12-month prospective study. Videosurgery Miniinv 2015; 10: 398-405.

17. Bužga M, Holéczy P, Švagera Z, et al. Effects of sleeve gastrectomy on parameters of lipid and glucose metabolism in obese women -6 months after operation. Videosurgery Miniinv 2013; 8: 22-8.

18. Pluskiewicz W, Buzga M, Holeczy P, et al. A comment on "Changes in bone mineral density in women following 1-year gastric bypass surgery" published by Casagrande DS et al. Obes Surg 2013; 23: 1885.

19. Adamczyk P, Buzga M, Holeczy P, et al. Body size, bone mineral density and body composition in obese women after laparoscopic sleeve gastrectomy: a 1-year longitudinal study. Horm Metab Res 2015; 47: 873-9.

20. Adamczyk P, Bužga M, Holéczy P, et al. Bone mineral density and body composition after laparoscopic sleeve gastrectomy in men: a short-term longitudinal study. Int J Surg 2015; 23: 101-7.

21. IPAQ. The IPAQ Group. Guidelines for Data Processing and Analysis of the International Physical Activity Questionnaire. Available from: http://www.ipaq.ki.se.

22. Bužga M, Marešová P, Petřeková K, et al. The efficacy of selected bariatric surgery methods on lipid and glucose metabolism: a retrospective 12-month study. Cent Eur J Public Health 2018; 26: 49-53.

23. Kasalický M, Bařinka A, Čierny M, et al. 10 years of sleeve gastrectomy in the Czech Republic in terms of the surgical procedure. Rozhl Chir 2016; 95: 425-31.

24. Bužga M, Hauptman K, Švagera Z, et al. Metabolic effects of sleeve gastrectomy and laparoscopic greater curvature plication: an 18-month prospective, observational, open-label study. Obes Surg 2017; 27: 3258-66.

25. Nicklas BJ, Penninx BWJH, Ryan A, et al. Visceral adipose tissue cutoffs associated with metabolic risk factors for coronary heart disease in women. Diabetes Care 2003; 26: 1413-20.

26. Brown JC, Harhay MO, Harhay MN. Anthropometrically-predicted visceral adipose tissue and mortality among men and women in the third national health and nutrition examination survey (NHANES III). Am J Hum Biol Off J Hum Biol Counc 2017; 29. doi: 10.1002/ajhb.22898.

27. Baumgartner R. Body composition in healthy aging. Ann N Y Acad Sci 2000; 904: 437-48.

Received: 17.12.2019, accepted: 10.02.2020. 